\title{
EL EMPRENDEDURISMO COMO POLÍTICA SOCIAL
}

\author{
Entrepreneurship as social policy
}

\author{
Alejandro Gabriel Mariatti Acosta ${ }^{1}$
}

La economía es el método. El objetivo es cambiar el alma

Margaret Thatcher

\begin{abstract}
Resumo
El siguiente artículo problematiza aspectos de la metamorfosis que atraviesa el mundo del trabajo y las consecuencias para la protección social contemporánea. Se aborda el pasaje o cambio de paradigma para comprender los nuevos diagnósticos sobre el desempleo, en particular sobre su origen y la responsabilidad en su resolución, colocando un profundo sentido neoliberal que tiene impactos en la modalidad y dispositivos para el abordaje, como política de protección social. En un primer momento se desarrollará el marco conceptual que problematizará el surgimiento del cambio de paradigma, con el que luego, en un segundo momento y a partir de este primer enfoque, se caracterizará la política pública de protección social y la atención al desempleo, tanto de programas de inserción laboral temporal, como de promoción al cooperativismo. Estos programas han sido seleccionados por su alcance nacional y por su carácter representativo por ser ésta una política del poder ejecutivo en Uruguay. En este sentido, sirve como ejemplo, para conocer el rumbo que desde el año 2005 le otorga, el gobierno progresista, a la atención al desempleo. La notoria incorporación del lenguaje propio del ethos empresarial en la retórica hacia la promoción del emprendedor desde la política social, para atender y responsabilizar en ese mismo acto al desempleado, es un camino que tiende a profundizar la desigualdad, naturalizar la lucha de clases, construir una explicación moralizante de la cuestión social y psicologizar de este modo los dispositivos para el abordaje.
\end{abstract}

Palabras clave: Política social; Activación; Emprendedurismo;

\begin{abstract}
The following article makes a critical analysis of central aspects of the current metamorphosis of the world of work and the consequences for contemporary social protection. It presents the idea of a paradigm shift is necessary to understand the new diagnoses on the phenomenon of unemployment. Particularly in regard to its origin and responsibilities in its resolution. In relation to this, it is noted that a deep neoliberal sense in the matrix of these elements have strongly impacts in the treatment modalities of social protection policies. At first, the conceptual framework will be developed from which the emergence of the paradigm shift has been problematized. Then, in a second moment, the public policy of social protection and the attention to unemployment resulting from both temporary labor insertion programs and promotion to cooperativism will be characterized and analized. These programs

\footnotetext{
${ }^{1}$ Doctor en Ciencias Sociales por la UdelaR/FCS. Magister y Licenciado en Trabajo Social, egresado de la UdelaR/FCS. Fue integrante de la Comisión de Ética Profesional de Trabajo Social en Uruguay (2013-2015), actualmente es integrante de la Comisión asesora en Investigación de FCS.
} 
have been selected for their national scope and to be representative of the policy on unemployment care of the progressive government in its fifteen-year management by the Executive Branch. The analysis shows an increase in a "business language", centered on the figure of "the entrepreneur" inside of the social policy. Language that simultaneously attends but holds the unemployed responsible for their situation. The article concluded that this paradigm shift constructs a moralizing explanation of the social question, psychologizes the public policy attention strategies, deepening social inequality and making class struggle invisible.

Keywords: Social policy ; Activation; Entrepreneurship;

\section{El cambio de paradigma}

La recesión mundial de los años setenta obligó al capital a recomponer la producción. Esa crisis, es parte de nuestra contemporaneidad. Las regulaciones como límites de protección a la industria nacional y el cuidado del empleo, los tipos de cambio diversificados y los subsidios, todas formas de regular la economía, fueron el centro del problema para el avance del orden civilizatorio impuesto por la agenda neoliberal, con consecuencias a escala planetaria. La ampliación de la ciudadanía se enfrentó con las exigencias de la rentabilidad mercantil nacida de la caída de la tasa de lucro. El informe de la Trilateralidad (CROZIER et al, 1975) realiza su diagnóstico de este impetu democratizador, como concesiones que han llegado a su punto culmine, luego de que pasaran las tres expansivas décadas (19401970) y se desacelerara el inédito crecimiento. La descalificación de todo lo público y estatal acompañara este deseo monetarista y liberal.

A desqualificação do Estado tem sido, como é notório, a pedra de toque do privatismo da ideologia neoliberal: a defesa do "Estado mínimo" pretende, funda mentalmente, o "Estado máximo para o capital"; nas palavras de Przeworski, constitui um projeto histórico da Direita, dirigido para liberar a acumulação [capitalista] de todas as cadeias impostas pela democracia" (NETTO, 2012, p. 422).

Para comenzar este análisis de trasformación del rol del estado y el alcance de lo público, se incorporan como fuente, las reflexiones de Anthony 
Giddens (2000) quien, en su preocupación gubernamental para asesorar al gobierno de Blair, establece una diferencia que le permite distinguir entre un tipo de política que es propia de un tipo de Estado anterior a ser superado, el "Estado de Bienestar Clásico" (EBC), diferenciándolo del nuevo rol del estado, el "Estado Social Inversor" (ESI). El primero regula al mercado de trabajo y promueve subsidios para situaciones de desempleo, pero eso lo vuelve más costoso. El segundo se aparta de esta primera actitud y apuesta a un abordaje en sentido contrario, con el fin de "activar" a la población para que esta se enfrente al mercado de trabajo, tratando de independizarlos del subsidio estatal que genera pasividad y comprometerlo a un compromiso personal.

El autor trabaja la idea de una transformación en el papel del estado en relación a la inversión en recursos humanos. “(...) un papel esencial que cumplir invirtiendo en los recursos humanos y la infraestructura requeridos para desarrollar una cultura empresarial" (GIDDENS, 2000, p. 119). E1 motivo de este viraje según Giddens (2000) está en lo que ha significado la transformación del mundo del trabajo, por dos razones fundamentales: la metamorfosis tecnológica que requiere nuevos niveles de especificidad en el conocimiento y el aumento en la esperanza de vida. Esto último estaría colapsando los seguros sociales tradicionales y el sistema mutual propio de los Estados de bienestar. Por estos motivos, el autor trae la idea de una asunción personal del riesgo responsablemente y el cultivo del "capital humano" (GIDDENS, 2000, pp. 120 y 121).

La idea está en el cambio de actitud del estado y las implicancias que esto tuvo para el alcance y ampliación de la ciudadanía (COUTINHO, 1997). La promoción en la asunción del riesgo y el alejamiento de un rol pasivo que solo atendía el problema, sin anticiparlo. La situación debe ser vista como una inversión previa con intenciones de modificar la actitud del ciudadano, valorado en términos de recurso y posibilidad de inversión. De esta forma se cultiva “(...) el desarrollo de un ethos empresarial responsable" (GIDDENS, 2000, p. 128), a nuestro juicio, en desmedro de la ciudadanía clásica. 
La educación atravesó centralmente la propuesta de Tony Blair, para quien su gobierno inglés tenía tres prioridades, “(...) educación, educación, educación ${ }^{2} "$ (GIDDENS, 2000, p. 130). Estas palabras son prácticamente iguales a las que expresó el presidente José Mujica en Uruguay al asumir la presidencia en $2010^{3}$. Según las interpretaciones del autor anglosajón, la dependencia del Estado pasivo, que otorga prestaciones, generaría un riesgo moral, "El riesgo moral existe cuando la gente utiliza la protección del seguro para cambiar su comportamiento, redefiniendo asi el riesgo para el que están asegurados" (GIDDENS, 2000, p. 136). Parece querer decir lo mismo que el "Camino de Servidumbre" al que se refiere Hayek (2006).

Una mirada resignada le permite entender que "El Estado de bienestar no está preparado para cubrir riesgos novedosos como los referentes al cambio tecnológico, la exclusión social o la creciente proporción de hogares monoparentales" (GIDDENS, 2000, p. 138). Del enfoque negativo de los seguros sociales que atienden las carencias se debe pasar al estado positivo. En este “(..) contribuyen los propios individuos y otros agentes además de la Administración" (GIDDENS, 2000, p. 139). Para Giddens, el bienestar no es un concepto exclusivamente económico, sino que “(...) deben ocuparse de promover mejoras psicológicas a la vez que económicas. (...) el asesoramiento, por ejemplo, puede ser en ocasiones más útil que el apoyo económico directo" (GIDDENS, 2000, p. 139).

El principio guía para el autor está en la inversión en "capital humano" donde sea posible. "En lugar de Estado de bienestar deberiamos colocar el Estado social inversor, funcionando en el contexto de una sociedad de bienestar positivo" (GIDDENS, 2000, p. 139). A su vez, las provisiones del bienestar deben integrarse con programas para el desarrollo activo de la sociedad civil. Como sintesis el autor identifica las características del estado social inversor con un gasto en "bienestar positivo", generado y distribuido no solo por el Estado sino por otros agentes, donde el foco principal está en

\footnotetext{
${ }^{2}$ Tony Blair en 1997 en el discurso en la Universidad de Southampton

${ }^{3}$ En este enlace se puede observar la asunción del Presidente José Mujica en 2010. https://www.youtube.com/watch?v=_yzbnHhB1NY
} 
expandir la responsabilidad individual, donde se promoverá la educación, la iniciativa y la autonomía.

Este despliegue consolida el pasaje de una visión tradicional sobre el desempleo, sobre el manejo y el control de tiempo del empleo y del desempleo. Se pasa de una concepción anclada en el principio negativo de no ociosidad a otro positivo de productividad (Ver ZANGARO, 2011, p. 39). El concepto de "activación” entonces será clave para entender dicho cambio.

En este nuevo modelo de intervención, basado en la activación, el principio articulador sería el fomento de la agencia individual (autonomía económica) para permitir una optimización de la posición individual en el mercado. Se lucha contra la "dependencia" de los sujetos, pero el objeto en torno al cual se problematiza la relación no es la dependencia del mercado sino la dependencia del Estado (CRESPO et al, 2009, p. 96)

"Activación" porque pretende una nueva actitud del participante, no desde la pasividad que promueve el subsidio, sino convocándolo con mayor protagonismo desde su entusiasmo por construir la solución, proponiéndole procesos de aprendizaje para el desarrollo de estrategias de presentación, búsqueda de empleo, participación en entrevistas de trabajo, diseño de "currículum vitae" y un infinito y kafkiano proceso de reconversión laboral.

Vale decir que en este proceso también intervienen privados, empresas donde su trabajo es buscar trabajo a desempleados que solicitan sus servicios. La orientación vocacional, los terapeutas de la ocupación, las empresas colocadoras, como “(...) industria del trabajo” (CALAMARI, 2010). “(...) la industrialización de la materia humana, de las capacidades cognitivas como principal sustento del trabajo" (CALAMARI, 2010, p. 90). Se refiere a la industria del trabajo como ese “(...) esfuerzo organizado e institucionalizado en pos de la capitalización y organización de un conjunto de dispositivos y saberes relacionados al trabajo" (CALAMARI, 2010, p. 94).

La valoración estatal sobre este nuevo desempeño del desempleado, se realiza para enfrentar los vaivenes de la economía contemporánea, flexible, global y cambiante, con la preocupación latente por el cuidado del clima de 
negocios y la inversión privada, donde la variable trabajo es un costo a ser reducido y donde el desempleo forma parte de la ecuación. Si bien el avance de la tecnología y los procedimientos hace que el desempleo sea sectorial, pudiendo haber pequeñas zonas de pleno empleo u otras donde la oferta y demanda no logran encontrarse, en términos generales, el desempleo tiende a colaborar con la contención de los salarios, la destrucción de sindicatos y espacios de negociación, entre otras cosas por el campo de batalla que abre por la competencia de los puestos de trabajo.

Dicha retorica vuelve sobre el sujeto una obligación de controlarse y de invertir en su "capital humano", con el afán de no ser excluido del proceso de producción, pues quedar fuera de este proceso, significa la muerte por inanición o la cárcel. Por lo tanto, es un poder que se ejerce sobre sí mismo, en los términos de Foucault, "tecnología del yo" (ZANGARO, 2011), que el individuo aplica sobre sí mismo. Para esta explicación neoclásica, la pobreza estaria vinculada directamente a la carencia de "capital humano", lo que refuerza la importancia del "paradigma de la activación" como solución individual y no estructural. Por consiguiente, esta responsabilización individual lleva a una explicación personal del fracaso y del control que debió haberse ejercido como "tecnología del yo".

El avance de esta perspectiva educativo-moralizante en torno a la explicación de la cuestión social expresa el nuevo ropaje neoconservador. La retórica económica incorporada al lenguaje cotidiano hace que sea común escuchar sobre la inversión en varios aspectos, salud, calidad de vida, educación, confort. La inversión, es propia de la nomenclatura del ethos empresarial (LÓPEZ RUIZ, 2007), y paso a ser parte de nuestra nomenclatura común. El sesgo moral que este enfoque tiene, supone la construcción diagnóstica del fracaso personal en el mercado como carencia cultural, actitudinal y educativa, una explicación neoconservadora que naturaliza al mercado como espacio de justicia siguiendo los parámetros del ideario neoliberal y coloca el fracaso como consecuencia de la irresponsabilidad personal, como explicación apologética (LUKÁCS, 2000). Invertir en "saber hacer" lo que el capital necesita y a la hora que lo necesita, 
podría ser una aproximación a medida de la utopía empresarial y el concepto de "capital humano".

Las determinaciones que reconstruimos en párrafos anteriores, significan un marco, para el desarrollo en Uruguay de programas que, paulatinamente fueron adscribiendo a este paradigma. Su desarrollo y fundamentación, está vinculado a la nación de capital humano y empleabilidad que desarrollare a continuación, para posteriormente ejemplificar a partir de caracterizaciones sobre tres diferentes programas, que se imbricación para su ejecución con los fundamentos de este nuevo paradigma en Uruguay.

\section{El capital humano y la empleabilidad}

El trabajador para algunos pensadores de finales del siglo XIX era un problema a conmensurar. En 1891, se publicó en el Reino Unido, un artículo de J.S Nicholson intitulado "El capital vivo del Reino Unido"4, que contenía en esencia, la necesidad que más adelante traerán los mentores del concepto de "capital humano" y otras denominaciones (Ver LÓPEZ RUIZ, 2007). El problema para Nicholson era encontrar el valor monetario de este capital vivo, como él mismo describe, “(..) de la misma forma en que sus pianos y cajas de pinturas lo son en el capital muerto (...) de la misma forma que los muebles de un stock inanimado" (LÓPEZ RUIZ, 2007, p. 404). En 1937 será el texto de F. V. Hayek "Economía y conocimiento" el que considerará el trabajo como un valor que potenciaría el crecimiento, siendo un antecedente directo de la teoría de "capital humano". (Ver PINO FREIRE et al, 2010, p. 105). T.W. Schultz, en 1959 hablará de modo más refinado, identificando este valor como "capital humano".

En junio de 1959 aparece publicado en The Social Service Review el artículo correspondiente a la conferencia dictada por Theodore W. Schultz (...) en la Universidad de Chicago. El título era provocativo, pero a la vez

\footnotetext{
${ }^{4}$ NICHOLSON. J. S. (1891), "The Living Capital of the United Kingdom", The Economic Journal, vol. 1, pp. $95-107$
} 
cauteloso: Investment in Man: An Economist's View" <en el hombre: la visión de un economista> (...) Schultz decía que se iba a "arriesgar a sugerir la hipótesis" de que las personas en sí mismas son una forma de riqueza y que esta riqueza no debe ser dejada de lado por el cálculo económico. Afirmaba: "los habitantes son, también, una parte importante de la riqueza de las naciones" cuya consideración viene siendo omitida por la economía. (LÓPEZ RUIZ, 2007, p. 400)

Sin embargo, mientras que para Nicholson se trata de un cálculo económico del "capital vivo", para el Schultz, será cuánto valen ciertas cualidades o habilidades en el mercado. Explica López Ruíz (2007) que, lo que para Nicholson es un costo, para Schultz es una inversión. Por eso realiza un paralelismo con reflexiones weberianas en torno a lo que significó la ética protestante y su disposición ascética al ahorro como proyección futura. En este caso, el ethos empresarial, convierte la vida en una empresa. Quien invierte en sí, está pensando en el futuro. La educación será vista como una inversión y desde 1960, fue notorio el aumento de literatura en el campo de la teoría de "capital humano" (LÓPEZ RUIZ, 2007).

Para López Ruíz, el inicio de esta reflexión que permite pensar 1o humano como capital se dio en 1960 y su lugar de nacimiento fue la Universidad de Chicago, de la que Schultz era el director desde 1946. Destinó gran parte de su investigación al desarrollo y la relación con los recursos humanos. "El ensayo sobre el valor económico de la educación” de Schultz obtuvo el premio nobel de economía en 1979. Para el autor, “(...) el trabajo es producto de una inversión, por lo tanto, no un medio de producción originario sino un medio de producción producido" (LÓPEZ RUIZ, 2007, p. 408). Detrás de esta reflexión del trabajador como un medio de producción producido, estaba Harry Johnson, economista canadiense que trabajó en la Universidad de Chicago desde 1959. La inversión en "capital humano", será la justificación para una explicación individual del empleo y una explicación del desempleo como fracaso personal, pero también para explicar el desarrollo frustrado de las economías dependientes, que, para Schultz, eran 
economías subdesarrolladas como consecuencia de su pobreza en "capital humano".

El desempleo de larga duración surgió de la ruptura del pleno empleo de las tres gloriosas décadas al que se hacía referencia con Hobsbawm (1999). Luego de la crisis de los años setenta, las posibilidades de acceder a un empleo, devienen en un incierto escenario, consolidando el problema como la responsabilidad en la inversión por educarse, que debe ser resuelto personalmente, una valoración individual y exclusiva sobre las propias habilidades, destrezas y posibilidades, de tener un empleo. Esto, es una medición traducida en "capital humano". Trabajadores como "(...) capitalistas de sus propias destrezas y habilidades" (LÓPEZ RUIZ, 2007, p. 410). Como explica el autor, en la era del inversor de "capital humano" independiente, se sugiere pensar a los trabajadores como trabajadoresinversores. Ya no son un costo sino inversores de su propio "capital humano". Ellos mismos ahora son un costo para sí. El ethos empresarial como forma de entender el mundo (weltanschauung), impondrá obligaciones de cómo vivir la vida.

La aceptación de un conjunto de atributos humanos como una forma de capital y como el resultado de una inversión impone a cada individuo un deber en relación a "su capital": cada individuo es responsable por las inversiones que hace o deja de hacer en sí mismo (LÓPEZ RUIZ, 2007, p. 415)

Ampliando las concepciones de Schultz, para Sen (1998, p. 69) el “(..) concepto se concentra en el carácter de agente de los seres humanos, que, por medio de sus habilidades, conocimientos y esfuerzos, aumentan las posibilidades de producción”. Sin embargo, repara en la debilidad que ésta interpretación tiene para medir lo humano, como reduccionismo y reivindica una ampliación del mismo, complejizándola en términos de “agencia" y entendida como un capital. El autor explica que el desarrollo del entorno favorece el desempeño de este "capital humano", ampliándolo como "capacidad humana". Capacidad del dialogo entre "agencia y entorno" de 
desarrollo social, como dialogo entre lo que porta el sujeto y el escenario. Se refiere de este modo a dicha limitación conceptual de "capital humano";

El concepto de capital humano es más limitado puesto que sólo concibe las cualidades humanas en su relación con el crecimiento económico mientras que el concepto de capacidades da énfasis a la expansión de la libertad humana para vivir el tipo de vida que la gente juzga valedera. Cuando se adopta esta visión más amplia, el proceso de desarrollo no puede verse simplemente como un incremento del PIB sino como la expansión de la capacidad humana para llevar una vida más libre y más digna. (SEN, 1998, p. 68)

Sen afirma que el concepto de "capacidad humana" centra su enfoque en la habilidad para llevar el tipo de vida que se considere valiosa y de ese modo incrementar sus posibilidades reales de elección. El escenario contemporáneo para la agencia, representa a inicio de siglo XXI, la recomposición de la tasa de lucro vía mundialización de la economía, con ampliación de los mercados y su lógica civilizatoria (DARDOT y LAVAL, 2009), acompañado de un salto tecnológico, con promoción de una metamorfosis sin precedentes en el mundo del trabajo, de tendencia a la precarización del empleo. El trabajo como capital variable es puesto en el centro de la recuperación de la tasa de lucro. Hacerlo más productivo, es hacerlo más barato y la ampliación del trabajo muerto vía automatización, permite la sustitución de mano de obra. El salto de la revolución informacional, como tercera revolución industrial, en el plano de la microelectrónica, especializa funciones, simplifica tareas y complejiza las relaciones de producción. Un escenario poco auspicioso y fértil para el despliegue de la agencia que rescata Sen.

Las formas de gestión del trabajo, constituyen un conjunto de prácticas por medio de las cuales el capital organiza la fuerza de trabajo. La aptitud emprendedora que Schumpeter (1976) creía privilegio de unos pocos, ahora es el espíritu global, convertido en la nueva racionalidad y difundido para todos como nuevo ethos. Un emprendedor es en lo que todos nos deberíamos de convertir. 
Foucault interpreto el, (...) nuevo arte de gobierno basado en la racionalidad de los propios agentes económicos. Pero ¿Quiénes son esos agentes económicos sino todos los individuos en el momento en que se ven a sí mismo (...) como una empresa? (LÓPEZ RUIZ, 2007, p. 422)

Este proceso tiene ida y vuelta en torno a la objetivación-subjetivación. Nos interesa aquí destacar esta excesiva preocupación sobre el individuo, y el avance de este recorte para explicar el fenómeno de desempleo de larga duración. Es un proceso ligado al ingreso de saberes que nacieron en la ciencia de la administración de empresas, desde involucramiento con la estadística a fines del siglo XIX (TOPALOV, 1990, p. 54). Con el correr del siglo XX, la visión del management impuso obligaciones heterodeterminadas, que se volverán auto-impuestas, entendido esto como proceso de desarrollo de una tecnología de producción-comunicación-poder (ZANGARO, 2010).

Los nuevos modelos de organización debilitaron algunos rígidos esquemas tayloristas-fordistas (ZANGARO, 2010) a escala planetaria. La tecnologización creciente y la movilidad financiera, plantearon nuevos usos productivos del trabajo. El avance de lo que se dio en llamar la sociedad del conocimiento, jerarquizó el lugar de las nuevas competencias relativas a procesos cognitivos, comunicativos y lingüísticos, capacidad de abstracción, herramientas intelectuales acordes al nuevo modelo de producción (ZANGARO, 2010).

Entre las competencias que requieren los empleadores en las economías tanto formales como informales de estas regiones en vías de desarrollo (además de las competencias básicas y técnicas) están la disposición a aprender; la aptitud para comunicarse, en forma tanto oral como escrita; los buenos hábitos de trabajo (puntualidad, aplicación, etc.); la capacidad de trabajar en equipo; la integridad personal; el liderazgo; el espíritu emprendedor, y la capacidad para el pensamiento analítico y crítico (OIT, 2014, p. 2) 
Competencia como palabra tiene dos raíces semánticas. Una que proviene del griego, y que hace referencia a la contienda, al encuentro por la victoria, a la competencia olímpica. La otra etimología, del latín, le otorga otro sentido, como responsabilidad sobre algo que le compete, que le es propio, para el que tiene competencia. El primero hace referencia a la competencia como contienda y el segundo supone una capacidad para algo. Ambas parecen estar presentes en este resurgir de la palabra competencia, pudiéndose sintetizar como saberes para la contienda.

Estos procesos de desarrollo, tienen la intención de captar la adhesión de los trabajadores por medios pacíficos, para mantener la subordinación. En algún punto, este despliegue de una mirada más singular, puede asociarse a procesos que promueven un mayor protagonismo personal, donde también hay espacio para las emociones y la creatividad sentimental del trabajador. Nada de esto lo exime de la concreta situación cosificante que en este vínculo se desarrolla. Explica Zangaro que en la literatura managerial este es el espíritu del capitalismo de nuestra época, que es la “(...) ideología que justifica el compromiso que los sujetos tienen con el capitalismo)" (2011, p. 21).

Este despliegue consolida el pasaje de una visión tradicional del manejo y el control de tiempo. Se pasa de una concepción anclada en el principio negativo de no ociosidad a otro positivo de productividad (Ver ZANGARO, 2011).

\section{Las políticas sociales con dimensión terapéutica}

Las politicas sociales y de atención al desempleo contemporáneas, para Crespo y Serrano (2013), se caracterizan por hacer una lectura del desempleo desde una mirada radicalmente psicologizante y moralizante. En el mismo instante en que “(..) apela a la responsabilidad personal, (...) desprovee al sujeto de las condiciones sociales y politicas para el ejercicio de la autonomía" (2013, p. 1111). Para los autores se pasa de la justicia a la terapia. El contrato social propio de la posguerra, sufre una transformación con el avance de la retórica neoliberal. Hay una transformación en el sentido 
de la solidaridad propia de la ciudadanía social, poniendo en cuestión algunas de sus características clásicas.

Hay en esta nueva orientación una intención de transformar los problemas socio-estructurales en problemas psicológicos. Hay sin dudas aquí una "batalla por el lenguaje" (Fairclough apud CRESPO Y SERRANO, 2013, p. 1112). La promoción de politicas de empleo incorpora conceptos como "empleabilidad, activación y flexiguridad" (CRESPO Y SERRANO, 2013, p. 1112). Esta última como una estabilidad-flexible, como un líquido-solido parafraseando a Bauman, ¿Será eso posible? El espacio se vuelve polisémico y ambiguo y entran allí todo un caleidoscópico reflejo de interpretaciones. Los fundamentos de esta retorica caminan por el lado de enfrentar a la pasividad con actividad, a la rigidez con flexibilidad y la dependencia con independencia. En este sentido, invierte la responsabilidad y supone un individuo que logra activarse, flexibilizarse e independizarse.

Hay una inversión del sentido, en tanto aquellos dispositivos que atendían la llamada cuestión social como conquistas colectivas para desmercantilizar esferas de la vida, ahora son entendidas como un problema que representa la dependencia y la pasividad, el camino de la servidumbre parafraseando a Hayek. Las nuevas intervenciones sobre el desempleo del "Estado terapéutico (...) gestiona la adaptación a las nuevas condiciones del mercado (...) la producción de un nuevo tipo de sujeto ciudadano" (CRESPO Y SERRANO, 2013, p. 1113). Se presenta el problema del desempleo como falta de "empleabilidad". Con este desplazamiento semántico, se hace del desempleo una patología de la voluntad. (CRESPO Y SERRANO, 2013). Para los autores, más que combatir el desempleo, se busca producir una forma de nombrarlo. Más que atender el problema, construyen el enfoque desde donde comprenderlo como problema. ¿'Son los desempleados actuales potenciales emprendedores realmente?

En este sentido, se importan una serie de rutinas y procedimientos desde el sector empresarial, supuestamente desde la neutralidad que la naturalización del mercado otorga, institucionalizando el proceso de 
reconversión semántica del problema del desempleo. Parece volver un siglo para atrás al colocar la voluntad, la pereza, el alcoholismo, propio de aquellas miradas caritativas, higienistas y moralizantes sobre "los malos pobres”. Se busca la no dependencia del Estado, sin reparar en que “(..) se invisibiliza, la dependencia vulnerabilizadora del mercado" (CRESPO Y SERRANO, 2013, p. 1115). Para los autores, este desplazamiento semántico posibilito un enfoque metonímico, entendiendo un todo por una de sus características singulares y de ese modo interpretar las secuelas del orden y el mercado de trabajo como un problema de "empleabilidad personal".

Este escenario de fatalidad como telón de fondo explicativo de una inercia inmodificable, funciona como paradigma para la atención individual, porque funciona como la otra salida posible, ya que la estructura se presenta incuestionable e inmodificable, el problema debe explicarse en otro lado. El avance de la automatización, en lugar de ser visto como triunfo de la ampliación del dominio humano sobre la naturaleza cada vez con menor esfuerzo, simplificando la tarea, generando tiempo libre, es visto de forma contraria, como el problema del desempleo que genera. El propio Banco Mundial y la Oxford Martin School en un estudio realizado recientemente, como proyección para el año 2030, explican que este fenómeno acorrala las economias. El informe proyecta que Etiopia tiene un $85 \%$ de riesgo de automatización de sus empleos, China un 77\%, Argentina un 65\% y Estados Unidos un 47\% (TAW, 2016) ${ }^{5}$. Esto consolida el desempleo estructural como una determinación mundial que avanza más rápido que las posibilidades de generar nuevos empleos. Esta inexorable ley parece querer ser atendida desde la promoción psicológica y el aumento de saberes individuales.

\section{La creciente presencia de la figura del emprendedor en la política de protección social en Uruguay}

\footnotetext{
${ }^{5}$ Basándose en el trabajo de Frey y Osborne, un reciente informe del Banco Mundial muestra que los países en desarrollo son altamente susceptibles a la automatización, incluso en relación con las economías avanzadas. (...) la susceptibilidad a la automatización a través de los países en desarrollo: Del 55\% en Uzbekistán, al 85\% en Etiopía, con una cuota sustancial de la mano de obra en alto riesgo de automatización en países como China y la India (77 por ciento y 69 por ciento, respectivamente). Estas cifras, a su vez, pueden ser puestas en comparación con el promedio de la OCDE, que el Banco Mundial estima en 57 por ciento (TAW, 2016, p. 18)
} 
En el año 2005 en Uruguay es creado el Ministerio de Desarrollo Social con la Ley 17866, con la competencia para coordinar las acciones y cubrir las "necesidades básicas" (sic) de quienes se encuentran en "indigencia o pobreza extrema" (sic), según describe el artículo 9 de dicha Ley (17866). Dentro del nuevo marco ministerial y además la ampliación de asistencia por medio de la asignación familiar, funcionarán una serie de programas que atenderán el desempleo. "La creación de ministerios sociales especializados en el tratamiento de situaciones vinculadas con la pobreza y con problemáticas específicas es otra de las medidas que también se extendieron en Latinoamérica". (MIDAGLIA et al ,2007, p. 469). Las nuevas secretarías son recomendación de entidades multilaterales o de financiación internacional. La creación de un Ministerio de Desarrollo para Uruguay era una deuda según las palabras de representante del BID en el año 2000,

(...) hay que agregarle que sucede ahora, que además de tener muchas instituciones públicas, tenemos instituciones que no son públicas involucradas en proyectos (...) quien es el que está responsabilizado de llevar adelante programas de este tipo (...) Hoy por hoy en Uruguay no existe un ámbito que tenga asignadas esas funciones, como por ejemplo un Ministerio Social (MIDAGLIA, 2001, p. 212).

Uno de los programas que traemos como ejemplo de una política social permeada por el nuevo paradigma de la activación y el emprendedurismo, es el programa “Trabajo por Uruguay” creado en 2005. El objetivo general del programa:

Contribuir al fortalecimiento ciudadano y a la mejora de la calidad de vida de los protagonistas del PANES ${ }^{6}$ mediante una experiencia sociolaboral que amplie oportunidades educativas y promueva el desarrollo de estrategias personales y sociales para la construcción de rutas de salida priorizando al trabajo como herramienta de inclusión social (MIDES, 2007, p. 17)

La preocupación anticipada de que el ciudadano desarrolle estrategias personales. En particular, el objetivo específico número cuatro establece “(...)

\footnotetext{
${ }^{6}$ Plan Nacional de Atención a la Emergencia Nacional 2005-2008
} 
el desarrollo de itinerarios personales orientados a la mejora del ingreso a través del fortalecimiento de las competencias laborales de autoempleo o empleo dependiente" (MIDES, 2007, p. 18). Es recurrente la búsqueda de resolución personal del conflicto entre el capital y el trabajo, incluso, desde la auto-explotación.

En aspectos metodológicos en el abordaje según el Ministerio, tuvo un rol destacado el llamado "acompañamiento”, “(..) El acompañamiento social representó la dimensión conceptual y metodológica que estructuró el Programa: vertebró toda la intervención, ya que se la considera clave para la promoción de procesos de cambio" (MIDES, 2007, p. 21). La dimensión personal era tan importante, que el documento explica que este acompañamiento debió realizarse “(...) brindando herramientas que puedan apropiarse a nivel personal, con orientaciones y estrategias diferentes para cada caso individual" (MIDES, 2007, p. 22). Sin dudas que el paradigma de la activación esta también expresando el paradigma de la individualización, esta preocupación por un abordaje a medida.

Se destaca que;

(..) una amplia mayoría de las $\mathrm{OSC}^{7}$ han realizado un acompañamiento personal, desplegando una diversidad de estrategias metodológicas (...) manteniendo el objetivo de atender cada situación personal, ajustando las herramientas y los tiempos a cada caso concreto (MIDES, 2007, p. 52).

Por la importancia de esta dimensión, el trabajo de ejecución tercerizada desde las Organización de la Sociedad Civil (OSC) se orientó en dos ejes: a) la formación en competencias socio laborales transversales traducidas como pautas y códigos culturales del mundo del trabajo y competencias específicas como destrezas y habilidades vinculadas a un área de actividad particular; b) “(...) el desarrollo de instancias individuales y colectivas como forma de reflexionar y aprender de las vicisitudes cotidianas del trabajo" (MIDES, 2007, p. 22). Hay una dimensión moral, que atraviesa la explicación del desempleo y permite el surgimiento de un dispositivo

\footnotetext{
${ }^{7}$ Organización de la Sociedad Civil
} 
terapéutico que atiende al desempleado como si adoleciera de un problema personal.

El concepto de empleabilidad, como una especie de suma de competencias y habilidades que el mercado estaría dispuesto a pagar, está presente, como valorando el capital humano, de ese trabajador devenido en empresa de sí mismo. "Se ha visto que en la medida que las y los protagonistas logran 'apropiarse de su destino' nuevamente, son capaces de tomar opciones para mejorar sus niveles de empleabilidad, (...)" (MIDES, 2007, p. 64). Queda claro que para el programa el foco del problema está en un asunto personal, fácilmente moralizarle. Expresa una concepción que psicológica la lucha de clases y la relación del capital con el trabajo.

La falta de un egreso con empleo, es un debe en la evaluación que el Ministerio realiza sobre el programa. "A pesar de que el programa no pretendia generar puestos de trabajo, la gran mayoría de las y los participantes muestran expresiones de insatisfacción porque Trabajo por Uruguay no les resolvió la cuestión del desempleo" (MIDES, 2007, p. 70)

En el año 2008, este programa es sustituido. Cuando se crea un nuevo plan nacional de protección social llamado "Plan de equidad" sustituyendo el plan anterior llamado "Plan de Emergentica" (PANES), es creado el programa "Uruguay Trabaja". Tiene nombre similar al anterior porque intenta realizar una transición sin interrupción.

Según describe el documento de Mides (2008), las acciones a desarrollar con este programa "Uruguay Trabaja” están sustentadas en tres pilares. a) el valor público de actividades que priorizan instituciones públicas; b) "Desarrollo de programas de promoción social a las y los trabajadores mediante una linea de intervención que atienda los factores personales y familiares que inhiben o dificultan el ingreso al mercado de trabajo (organización familiar, violencia doméstica, conductas adictivas, problemas de salud, ejercicio de derechos" (MIDES, 2008, p. 59); c) Desarrollo de programas de capacitación laboral. 
Queda definido con la Ley 18240 y el decreto 222/008 que la reglamenta, el programa "Uruguay Trabaja", que oferta un promedio de 3.000 puestos de empleo transitorio por año de 30 horas semanales durante 9 meses con "Acompañamiento social: personal, familiar y colectivo para el desarrollo de procesos de integración social al mundo del trabajo" (MIDES, 2008, p. 59) La evaluación realizada en 2011, valora el impacto que el programa tiene en la población sobre la que se focaliza, resaltando el valor de los componentes de "activación", tales como las nuevas habilidades, conocimientos y la mejora de su autoestima, continuando una línea de trabajo que viene siendo desarrollada desde "Trabajo por Uruguay".

(...) puede afirmarse la permanencia de ciertos resultados de efecto inmediato en los participantes ya constatados en anteriores evaluaciones de Programas Mides (Trabajo por Uruguay, Rutas de Salida, Uruguay Integra, etc.) como ser: la incorporación de nuevas habilidades por parte de los participantes de potencial importancia para su inclusión económica (un oficio, la mejora en el manejo de la informática, etc.), como para su inclusión social (mejora en su autopercepción y autoestima, ampliación de sus redes vinculares más próximas, aumento en la participación en organizaciones fundamentalmente entre aquellos que afirmaban no participar de ningún espacio social, etc.) (MIDES, 2011, p. 102)

E1 programa "Uruguay Trabaja", cuenta para el último mes de ejecución, la incorporación de un técnico que realiza una tarea muy particular. Es contratado desde la figura de "técnico en inserción laboral" y atiende a los usuarios del programa con el objetivo de dar un último tratamiento a su desempleo. Como si representara el toque final de una obra de arte o el impulso más sofisticado del programa, aparece en el epilogo, como para frotar una lámpara para que surja un genio este "técnico en inserción laboral", que tiene la tarea de lograr que aparezca de ese mismo mercado que expulso al trabajador, una nueva propuesta de trabajo. El técnico, acompaña al usuario para que este mejore su actitud frente a su desempleo, con técnicas que mejoran su apariencia y ayudan a sistematizar información del mercado de trabajo. También le enseñara a mejorar su curriculum vitae y el manejo de su conducta en entrevistas y en la propia búsqueda de trabajo, mejorando sus técnicas. Es un manual para que el 
desempleado comprenda en primer lugar que su desgracia nada tiene que ver con una situación histórica producto de la lucha de clases, sino por el contario, de una situación biográfica de la que debe hacerse responsable individualmente, con todo el peso de la moralidad.

El rol del "coaching laboral" promueve la inversión laboral personal. Para este técnico en inserción laboral su abordaje se centra en la motivación a los desempleados, con la intención de influir en los comportamientos personales, en su búsqueda de empleo y en la promoción de una actitud perseverante del usuario, suponiendo o diagnosticando el fracaso como consecuencia de su inmadurez personal y emocional.

Ha sido impulsado en estos años y por medio de este Ministerio el cooperativismo como expresión progresista del emprendedurismo. En el año 2008, el Estado con la intención de promover políticas públicas orientadas al sector cooperativismo, creará el Instituto Nacional de Cooperativismo (INACOOP) con la Ley 18.407, artículo 186. Sus cometidos van en esa dirección, vinculándose con la sociedad civil, promocionando los valores cooperativos y formulando planes de desarrollo y evaluaciones del sector y su relación con la economía en general. En 2015 con la Ley 19337, se crea el Fondo de Desarrollo (FONDES) destinado a financiar micro emprendimientos y se lo vincula con INACOOP para poder asistir a las cooperativas creadas. Con esta vinculación se le intenta dar mayor dinamismo a la financiación de emprendimientos cooperativos. Una mitad del FONDES es administrado por el INACOOP.

En el artículo 13 de esta Ley 19337, se establece que

La Junta Directiva del FONDES INACOOP tendrá como cometidos principales, adicionales a los establecidos en el artículo $3^{\circ}$ de la presente ley, los siguientes: A) Promover y apoyar el desarrollo de las distintas formas de la economía social y solidaria, y en particular, las previstas en los siguientes literales. B) Promover y apoyar el desarrollo de las empresas cooperativas reguladas por la Ley $\mathrm{N}^{\circ} 18.407$, de 24 de octubre de 2008. 
En 2017, Mides firma un convenio con INACOOP, para financiar emprendedores y cooperativistas vinculados al reciclaje.

El Ministerio de Desarrollo Social transferirá tres millones de pesos al Instituto Nacional de Cooperativismo, tras la firma de un convenio para la formación y capacitación de cooperativas de clasificadores de Bella Unión, Salto, Paysandú, Paso de los Toros y Canelones y de próximos grupos en Rivera, Flores, Maldonado y Rocha. El objetivo es promover la creación, el fortalecimiento y la autogestión de los emprendimientos (PRESIDENCIA, 2017)

En ese mismo acto, la Directora de la DINESIL evidenció la intención de que las cooperativas, creadas en un marco de protección o regulación estatal, ahora, puedan valerse por sí mismas, es decir, activarse en relación a la no dependencia del Estado y asumir la relación con el mercado. La financiación iría para promover la capacitación tendiente a fortalecer esta independencia. La "cultura de la protección” debe ser "activada".

En este proceso se desprende la importancia que tiene para atender el desempleo de larga duración desde el MIDES, la figura del cooperativista, como emprendedor monotributista. La retórica del ethos empresarial, adaptadas para el público que accede a estos programas. La vieja figura del cooperativismo, como tradición desde los pioneros de Rocherdale en el siglo XIX, ahora deviene como estrategia de abordaje de la llamada cuestión social, con el ropaje progresista, porque son propuestas en el marco del gobierno de la izquierda en Uruguay, de una política focalizada de "combate a la pobreza" con intenciones de independizar del apoyo estatal a los beneficiarios para convertirlos en empresarios monotributistas.

A partir de esta ejemplificación desde programas específicos, se ha tratado de demostrar las implicancias de este nuevo paradigma para con la protección social en Uruguay, particularmente en los años que significaron tres lustros de gobierno "progresista". En este sentido, parece sustancioso, dar cuenta de las complejidades que entrañan estos procesos, como necesidad de encontrar peculiaridades que expliquen con mayor detenimiento estos procesos. 


\section{Conclusiones}

Como explicaba Marx, el trabajador está involucrado en un proceso de constante elastización de su fuerza de trabajo para aumentar la producción de mercaderías y sostener la tasa de ganancia. Tres revoluciones multiplicaron este proceso. Primero, la revolución de la máquina de vapor en el siglo XVIII y con ella el motor de transmisión, con lo cual una máquina herramienta pasaba a comandar el proceso de trabajo; luego la segunda revolución del motor eléctrico en el siglo XIX; finalmente el descubrimiento casi al unísono de la energía nuclear y el desarrollo de la microelectrónica. A través de este proceso, el trabajador y su "capital humano" acompañan el desarrollo, donde cada vez más el lugar protagónico y dominante es el de la automatización. El avance de la precarización, la flexibilización y el desempleo de larga duración han permitido el avance sobre el trabajo, consolidando el triunfo de un paradigma que promueve su atomización.

El trabajador que no logre la adaptación a este nuevo escenario, será patologizado. Como se pudo demostrar, el "paradigma de la activación" como promoción del "empoderamiento de la agencia", es funcional con propuestas tanto de derecha como de izquierda, pues ambas promueven la participación y la responsabilización individual, como si el neoliberalismo los hubiera permeado a ambos y se pudieran encontrar juntos para reflexionar sobre este tema. El mercado parece oficiar de ámbito neutral, donde hay que generar oportunidades. La difusión a escala planetaria del capitalismo, sobre todo luego de 1992 con la caída del bloque de socialismo real, promovió el triunfo casi sin adversarios de una subjetividad empresarial, con valores de responsabilidad e individualismo y doctrinas de la administración privada.

El emprendedor, como acentuación de la individualidad, significó la imagen del expansionismo norteamericano y el "mito internacional de vaquero" (HOBSBWAM, 2013). El contrato que antes era sinónimo de seguridad ahora se vuelve arena movediza, "La flexibilidad va a aparecer como una exigencia totalizante, (...) la regulación politica del contrato laboral, 
que ha constituido uno de los ejes de la protección (...) se convierte, paradójicamente, en su contrario, en la causa de su vulnerabilidad" (CRESPO y SERRANO, 2012, p. 40).

Los pilares de este nuevo orden lucharán contra la dependencia de los ciudadanos para con el Estado, promoviendo la responsabilidad individual sobre la apropiación de mercantilizados saberes llamados "capital humano" y que explica el "nivel de empleabilidad". Lo patológico será para quienes no superen la dependencia, valuando su actitud o voluntad frente a este problema personal. El triunfo neoliberal se ejemplifica cuando se hace evidente la intención de mercantilizar todas las esferas de la relación social y todas las acciones humanas. Todo se convierte en objeto de esta visión maximizadora de beneficios. El sujeto debe aprender a venderse, a conocer sus fortalezas y debilidades, conocer sus emociones y controlarlas, saber mirarse al espejo y medirse con la regla del mercado y poner en juego su capital, como inversión para la mejora de su "nivel de empleabilidad", viendo donde hay una oportunidad de lucro para la inversión de su capital, de su sí mismo que es su empresa.

Es claro el avance de una lógica empresarial como describe López Ruiz (2007) en el escenario cotidiano. Su presencia, fortalece la idea de una "justicia natural" dada por el supuesto del mercado como espacio de encuentro entre libres capitales. Dardot y Laval (2009), encuentran una línea de continuidad que, habiendo nacido en la construcción del mercado y tomando como norma la competencia y ésta luego, como norma de la actividad de los agentes económicos, de la construcción del Estado y de su acción, llegan finalmente, hasta la competencia como norma de la conducta del sujeto-empresa. Estos representan momentos distintos por los que se produce la extensión de la racionalidad mercantil a todas las esferas de la existencia humana. Esto hace para los autores a la razón neoliberal, una verdadera razón-mundo.

Esta nueva razón del mundo se cuela por los intersticios de las políticas de protección social y atención al desempleo en Uruguay, como siguiendo las recomendaciones del "Estado social inversor" que recomienda 
Giddens (2000). Aquellas interpretaciones sobre la cuestión social a partir de trayectorias biográficas personales, fueron parte del repertorio que atendió el desempleo desde una preocupación por "activar" el espíritu adormecido de los usuarios de los programas. Coloca en el sujeto la responsabilidad cuando lo convoca para mejorar su empleabilidad con la orientación de un técnico en inserción laboral, colaborando con una construcción reificada de los problemas de la sociedad capitalista. El caso Uruguay, presenta por medio del emprendedor cooperativista que parece representar un hibrido entre el empresario y el cooperativista, una tendencia "activadora". Las características de los programas y su énfasis en las competencias laborales tanto en Trabajo por Uruguay y Uruguay Trabajo, expresan la dimensión singular del abordaje, lo que permite un tratamiento moralizante de la cuestión social y disciplinante (ZANGARO, 2011).

Es el triunfo de una ética empresarial que toma por asalto la vida cotidiana y convierte al individuo en una empresa. Las relaciones sociales pasan a ser ordenadas según el modelo de mercado en el capitalismo del siglo XXI. Como explican Dardot y Laval (2009), el neoliberalismo antes que ser una ideología o una política económica, es más bien una nueva racionalidad donde la lógica del mercado es llevada hasta los rincones más íntimos del ser. Es un orden civilizatorio que está construyendo al ser humano neoliberal, integramente sumergido en la competición mundial. Este individualismo moderno, comparte la doble dimensión de ciudadano con derechos y a la vez con intereses económicos, sin embargo, para los autores, en la disputa entre este hombre ciudadano y el hombre útil al interés económico, el primero sucumbe ante el segundo. Para los autores, las relaciones humanas serán sometidas a la regla del máximo provecho.

Como indican Dardot y Laval (2009), el sujeto es unificado en un sentido empresarial, perdiendo la pluralidad de la democracia liberal, producidos como sujetos emprendedores que, refuerzan las relaciones de competición entre sí. Este es el proyecto del neo sujeto en el neoliberalismo (DARDOT y LAVAL, 2009), como apología del orden. La racionalidad 
empresarial articula todas las relaciones de poder en la trama de un mismo discurso bajo el estandarte de la ética del emprendedor y el ayúdate a ti mismo. El sujeto es condenado al proceso mismo de mejora de sí y perfeccionamiento sin cesar de sus resultados y sus rendimientos.

Como indica Baqués Quesada (2002), el gran reto que se le plantea al neoconservadurismo es, reconstruir de una vez por todas una filosofia adecuada a la buena marcha de la economía de mercado y sus exigencias en el terreno de la moral. La responsabilización del desempleado y el trabajo sobre su actitud parecen ser parte de la respuesta a este reto neoconservador, de "cambiar el alma".

\section{Bibliografía}

BASQUÉS QUESADA Josep. La ilustración escocesa: ¿un depósito de intuiciones para el neoconservadurismo? Revista Estudios politicos (Nueva Época) Núm. 118. Octubre - diciembre 2002. Disponible en: file:/ / C:/Users/Pc/Downloads /Dialnet-LaIlustracionEscocesa-287609.pdf

CALAMARI. Andrea. Del trabajador al empleable. Una mirada sobre el discurso de la industria laboral. Tesis Doctoral. Universidad de Nacional de Rosario. Inédito. 2010

COUTINHO. Carlos Nelson. Notas sobre ciudadanía y modernidad. Revista Praia Vermelha. Estudios de Politica e Teoria Social. V1 $\mathbf{N}^{\circ} 1$. Rio de Janeiro. 1997.

CRESPO. Eduardo y SERRANO. Amparo. Las paradojas de las políticas de empleo europeas: de la justicia a la terapia. Revista Universidad Psychologica, 12 (4), 1111-1124 Doi: 10/11144Javeriana. USPY12-4. ppee. 2013. Disponible en: https:/ / bit.ly/2YooV4e

CRESPO. Eduardo y SERRANO. Amparo La psicologización del trabajo: la desregulación del trabajo y el gobierno de las voluntades. En Revista Teoria y critica de la psicologia. V 2. Pp 33-48. 2012. Disponible en: https:/ / dialnet.unirioja.es / servlet/articulo?codigo $=5895477$

CRESPO. Eduardo, REVILA. Juan Carlos, SERRANO. Amparo. Del Gobierno del trabajo al gobierno de las voluntades: El caso de la activación. Editorial Psicoperspectivas. ISSN 0718-6924 VOL. VIII, No 2. 2009. Disponible en: http://www.psicoperspectivas.cl/index.php/psicoperspectivas/issue/view/1 0 
CROZIER. Michel, HUNTINGTON. Samuel y WATANUKI. Joji. The crisis of democracia. The Trilateral Commission. New York University PressEd. Library of Congress Catalog Card Numeber: 75-27167 ISBN 0-8147-1364-5. 1975.

DARDOT. Pierre y LAVAL. Christian. La nueva razón del mundo. Ensayo sobre la sociedad neoliberal. Ed Editorial Gedisa Calves contemporáneas. Barcelona. 2009.

FOUCAULT. Michel. Tecnologias del yo y otros textos afines. Ed Paidos Bs As. 2008

GIDDENS. Anthony. La tercera via. La renovación de la socialdemocracia. Ed. Taurus. Argentina. 2000

HAYEK. Frederick Van. Camino de servidumbre. Alianza Editorial, Madrid. 2006

HOSBSBAWM. Erich. Un tiempo de rupturas. Crítica. Bs As. 2013

LÓPEZ RUIZ. Osvaldo. "Ethos empresarial: el <capital humano> como valor social. Revista Estudios sociológicos, Vol. XXV No 74 pp 399-425. México. 2007

LUKÁCS. George. La crisis de la filosofia Burguesa. Copyright. www.elaleph.com 2000

MIDAGLIA. Carmen; ANTÍA. Florencia. "¿Una nueva síntesis? La agenda social de la izquierda uruguaya a dos años de gobierno". Cuaderno CRH, Salvador V20 $\mathrm{n}^{\circ}$ 51. P 463-478. 2007. Disponible en: http://www.scielo.br/scielo.php?script=sci_arttext\&pid=S010349792007000300007

MIDAGLIA. Carmen. Los dilemas de la colaboración público privada en la provisión de servicios sociales. Pp 203-223. En CALAME. P y TALAMANT. A. Con el Estado en el corazón. Ed. Trilce. Montevideo. 2001

MIDES. Programa Uruguay Trabaja Edición 2009. Resultados de la Evaluación. Informe Final. 2011. Disponible en: http:/ / dinem.mides.gub.uy/innovaportal/file/61588/1/uruguay-trabaja.informe-de-resultados.-encuesta-pannel.-2009.pdf

MIDES. Plan de equidad $3^{\circ}$ Edición. IMPO. Montevideo. 2008

MIDES. Sistematización del programa Trabajo por Uruguay. "Del vientre de los sueños nace la realidad". Mides 2007 
NETTO. José Paulo. "Crise do capital e consequencias societárias". Sao Paulo. Revista Servico Social e Sociedade N ${ }^{\circ} 111.2012$

OIT. Competencias para el empleo. Orientaciones de politicas. 2014. Disponible en: file:///C:/Users/Pc/Downloads/PB-EMP-SK-blue-SYouth_web.pdf

PINO FREYRE. Romelia y PEDRERO GONZÁLEZ. Edwin. “Carlos Marx y el capital... ¿humano?” en Revista Internacional Marx Ahora. La Habana. pp $103-121.2010$

PRESIDENCIA DE LA REPUBLICA (2017). Disponible en: https://www.presidencia.gub.uy/comunicacion/comunicacionnoticias/mide s-inacoop-convenio-cooperativas-de-clasificacion-de-residuos

SCHUMPETER. Joseph. Teoria del desenvolvimiento económico: una investigación sobre ganancias, capital, crédito, interés y ciclo económico. FCS México. 1976

SEN. Amartya. Capital humano y capacidad humana. En revista Cuadernos de economia. Volumen 17, Número 29, p. 67-72. Santa Fe de Bogotá. ISSN electrónico 2248-4337. ISSN impreso 0121-4772. 1998 Disponible en: https://dialnet.unirioja.es / servlet/articulo?codigo=4934956

TAW. TECHNOLOGY AT WORK v2.0 The Future Is Not What It Used to Be. Citi GPS: Global Perspectives \& Solutions. January 2016. 2016. Disponible en: http://www.oxfordmartin.ox.ac.uk/downloads/reports/Citi_GPS_Technolog y_Work_2.pdf

TOPALOV. Christian. "De la "cuestión social" a los "problemas urbanos": Los reformadores y la población de las metrópolis a principios de siglo XX" (Reproducido con la autorización de la UNESCO, del original publicado en la Revista Internacional de Ciencias Sociales, $N^{\circ} 125$, PP. 41-71 septiembre. 1990

ZANGARO. Marcela. Subjetividad y trabajo. Una lectura foucaultiana del managment. Ed. Herramienta. Bs As. 2011

ZANGARO. Marecla. El managment en el cruce de la subjetividad y el trabajo. Revista Herramienta N ${ }^{\circ}$ 44. Bs As. 2010

LEGISLACIÓN NACIONAL DE URUGUAY:

17.866 creación del programa Trabajo por Uruguay https:/ / legislativo.parlamento.gub.uy/temporales /leytemp2267686.htm

18.240 creación del programa Uruguay Trabaja https://legislativo.parlamento.gub.uy/temporales/leytemp2414406.htm 
El emprendedurismo como política social | Alejandro Gabriel Mariatti Acosta

18.407 creación del Instituto Nacional de Cooperativas https:/ / www.impo.com.uy/bases/leyes / 18407-2008

19.337 creación del Fondo Nacional de Desarrollo https://www.impo.com.uy/bases/leyes/19337-2015 